\title{
Number of cerebral lesions predicts freedom from new brain metastases after radiosurgery alone in lung cancer patients
}

\author{
DIRK RADES ${ }^{1,2}$, STEFAN HUTTENLOCHER ${ }^{1}$, MAI TRONG KHOA ${ }^{2,3}$, PHAM VAN THAI ${ }^{3}$, \\ DAGMAR HORNUNG ${ }^{4}$ and STEVEN E. SCHILD ${ }^{5}$
}

\author{
${ }^{1}$ Department of Radiation Oncology, University of Lübeck, Lübeck 23538, Germany; ${ }^{2}$ Nuclear Medicine Department, \\ Hanoi Medical University; ${ }^{3}$ Nuclear Medicine and Oncology Center, Bach Mai Hospital, Hanoi 10000, \\ Vietnam; ${ }^{4}$ Department of Radiation Oncology, University Medical Center Eppendorf, Hamburg 20246, \\ Germany; ${ }^{5}$ Department of Radiation Oncology, Mayo Clinic, Scottsdale, AZ 85259, USA
}

Received September 5, 2014; Accepted April 29, 2015

DOI: $10.3892 / \mathrm{ol} .2015 .3370$

\begin{abstract}
Numerous patients with few brain metastases receive radiosurgery, either alone or in combination with whole-brain irradiation. The addition of whole-brain irradiation to radiosurgery reduces the rate of intracerebral failures, particularly the development of new cerebral lesions distant from those treated with radiosurgery. Less intracerebral failures mean less neurocognitive deficits. However, whole-brain irradiation itself may lead to a decline in neurocognitive functions. Therefore, a number of physicians have reservations with regard to adding whole-brain irradiation to radiosurgery. Prognostic factors that allow an estimation of the risk of developing new cerebral metastases can facilitate the decision regarding additional whole-brain irradiation. Since primary tumors show a different biology and clinical course, prognostic factors should be identified separately for each primary tumor leading to brain metastasis. The present study investigated 10 characteristics in a series of 98 patients receiving radiosurgery alone for 1-2 cerebral metastases from lung cancer, the most common primary tumor associated with brain metastasis. These characteristics included radiosurgery dose, age, gender, performance status, histology, number of cerebral lesions, maximum total diameter of cerebral lesions, main location of cerebral lesions, extracranial spread and interval from first diagnosis of lung cancer to administration of radiosurgery. On univariate analysis, the number of cerebral lesions prior to radiosurgery ( 1 vs. 2 lesions) was the only characteristic significantly associated with freedom from new brain metastases $(\mathrm{P}=0.002)$. In cases of 2 lesions, $73 \%$ of patients developed new cerebral lesions within 1 year. On multivariate analysis, the number of brain metastases remained significant
\end{abstract}

Correspondence to: Professor Dirk Rades, Department of Radiation Oncology, University of Lübeck, Building 40, 160 Ratzeburger Allee, Lübeck 23538, Germany

E-mail: rades.dirk@gmx.net

Key words: lung cancer, new brain metastases, radiosurgery, whole-brain irradiation, number of cerebral lesions (risk ratio, 2.46; 95\% confidence interval, 1.34-4.58; $\mathrm{P}=0.004$ ). Given the high rates of new cerebral lesions in patients with 2 brain metastases, these patients should be strongly considered for additional whole-brain irradiation.

\section{Introduction}

Numerous patients with few brain metastases receive neurosurgical resection or radiosurgery, either alone or in combination with whole-brain irradiation. As radiosurgery is similarly effective but less invasive than resection, the use of radiosurgery for the treatment of brain metastases has become more popular (1-3). Previous studies have shown that the addition of whole-brain irradiation can improve intracerebral control when compared with radiosurgery alone $(4,5)$. As an intracerebral recurrence has been reported to be a major cause of neurocognitive deficits, the addition of whole-brain irradiation, which reduces the risk of intracerebral failure, also appears beneficial for the patients from this viewpoint $(6,7)$. However, whole-brain irradiation itself can lead to a decline in neurocognitive function. This important treatment-related late morbidity was found to occur significantly more frequently after radiosurgery plus wholebrain irradiation than after radiosurgery alone at 4 months in a randomized trial of 58 patients with brain metastases from different primary tumors (8). Therefore, radiation oncologists often have reservations with regard to administering wholebrain radiotherapy in addition to radiosurgery (8). As new brain metastases distant from those treated with radiosurgery are the major cause of intracerebral failure, the decision for or against the addition of whole-brain irradiation would be facilitated if the risk of developing such new intra-cerebral lesions could be estimated (4). In order to achieve this goal, knowledge of significant predictors of the risk of developing new brain metastases is mandatory. As the primary tumors leading to brain metastases vary considerably with respect to their biology and course of disease, prognostic factors must be separately identified for each primary tumor associated with metastases to the brain. Of these primary tumors, lung cancer is the most common $(\sim 50 \%)$. Therefore, the present study focuses on patients receiving radiosurgery alone for a small number of cerebral metastases from lung cancer. The major goal of the study was the identification 
Table I. Univariate analysis of freedom from new brain metastases.

\begin{tabular}{|c|c|c|c|c|}
\hline \multirow[b]{2}{*}{ Parameter } & \multicolumn{3}{|c|}{ Freedom from new brain metastases, $\%$} & \multirow[b]{2}{*}{ P-value } \\
\hline & At 6 months & At 12 months & At 24 months & \\
\hline \multicolumn{5}{|l|}{ Radiosurgery dose, Gy } \\
\hline$<20(\mathrm{n}=38)$ & 59 & 52 & 46 & \\
\hline$\geq 20(n=60)$ & 75 & 45 & 28 & 0.98 \\
\hline \multicolumn{5}{|l|}{ Age, years } \\
\hline$\leq 59(\mathrm{n}=49)$ & 70 & 44 & 39 & \\
\hline$\geq 60(\mathrm{n}=49)$ & 67 & 54 & 34 & 0.54 \\
\hline \multicolumn{5}{|l|}{ Gender } \\
\hline Female $(\mathrm{n}=47)$ & 73 & 49 & 25 & \\
\hline Male $(\mathrm{n}=51)$ & 63 & 48 & 43 & 0.99 \\
\hline \multicolumn{5}{|l|}{ ECOG performance score } \\
\hline $0-1(n=64)$ & 70 & 54 & 43 & \\
\hline $2(n=34)$ & 64 & 33 & 16 & 0.16 \\
\hline \multicolumn{5}{|l|}{ Histology } \\
\hline Adenocarcinoma $(\mathrm{n}=62)$ & 67 & 42 & 23 & \\
\hline Others $(n=36)$ & 70 & 63 & 53 & 0.23 \\
\hline \multicolumn{5}{|l|}{ Number of cerebral lesions } \\
\hline $1(\mathrm{n}=61)$ & 73 & 62 & 41 & \\
\hline $2(n=37)$ & 60 & 27 & 15 & $<0.01$ \\
\hline \multicolumn{5}{|c|}{ Max. diameter of cerebral lesions, $\mathrm{mm}$} \\
\hline$\leq 18(\mathrm{n}=50)$ & 75 & 53 & 44 & \\
\hline$>18(\mathrm{n}=48)$ & 60 & 43 & 19 & 0.11 \\
\hline \multicolumn{5}{|c|}{ Main location of cerebral lesions } \\
\hline Supratentorial $(\mathrm{n}=84)$ & 68 & 46 & 25 & \\
\hline Infratentorial $(\mathrm{n}=14)$ & 71 & 71 & 71 & 0.19 \\
\hline \multicolumn{5}{|l|}{ Extracranial spread } \\
\hline No $(n=64)$ & 74 & 50 & 40 & \\
\hline Yes $(n=34)$ & 57 & 49 & 0 & 0.19 \\
\hline \multicolumn{5}{|c|}{$\begin{array}{l}\text { Interval from lung cancer diagnosis to } \\
\text { radiosurgery, months }\end{array}$} \\
\hline$\leq 11(\mathrm{n}=51)$ & 79 & 49 & 44 & \\
\hline$\geq 12(n=47)$ & 58 & 47 & 23 & 0.31 \\
\hline
\end{tabular}

of independent predictors regarding the development of new cerebral metastases in this particular group of cancer patients.

\section{Patients and methods}

Patients. A total of 98 patients receiving radiosurgery alone for 1-2 brain metastases of $>4 \mathrm{~cm}$ in size from lung cancer at the University of Lübeck (Lübeck, Germany) or the University Medical Center Eppendorf (Hamburg, Germany) between January 2000 and 2014, were retrospectively analyzed with respect to freedom from new cerebral lesions. All patients were diagnosed with stage IV lung cancer, according to the American Joint Committee on Cancer staging system (9). The present study was approved by the ethics committee of the University of Lübeck
(Lübeck, Germany; reference no. 13-038A and 14-273A). Radiosurgery was performed with photon beams from a linear accelerator (Siemens Medical Systems, Concord, CA, USA; Varian Medical Systems, Palo Alto, CA, USA).

Characteristics. A total of 10 characteristics were evaluated for associations with freedom from new brain metastases. These characteristics consisted of the radiosurgery dose ( $<20$ vs. $\geq 20 \mathrm{~Gy}$; doses prescribed to the $80-90 \%$ isodose level) (10), age ( $\leq 59$ vs. $\geq 60$ years; median age, 59 years), gender, Eastern Cooperative Oncology Group performance score (0-1 vs. 2), histology (adenocarcinoma vs. others), number of cerebral lesions (1 vs. 2), maximum total diameter of all cerebral lesions ( $\leq 18$ vs. $>18 \mathrm{~mm}$; median, $18 \mathrm{~mm}$ ), main location of 
the cerebral lesions (supratentorial vs. infratentorial), extracranial spread (no vs. yes), and the interval between the first diagnosis of lung cancer and the administration of radiosurgery ( $\leq 11$ vs. $\geq 12$ months; median interval, 11 months).

Statistical analysis. For the univariate analysis of freedom from new cerebral lesions, the Kaplan-Meier method and the log-rank test were used (11). Characteristics that achieved significance were additionally analyzed in a multivariate manner with the Cox proportional hazards regression analysis. The statistical analyses were performed with the JMP software, version 10 (SAS Institute Inc., Cary, NC, USA). P<0.05 was used to indicate a statistically significant difference.

\section{Results}

The results of the univariate analysis of freedom from new cerebral lesions are shown in Table I. The only characteristic that was found to be significantly associated with freedom from new brain metastases was the number of cerebral lesions prior to radiosurgery $(\mathrm{P}=0.002)$. If 2 brain metastases were present, only 27 and $15 \%$ of patients were free from new cerebral lesions at 12 and 24 months post-radiosurgery, respectively. According to the subsequent multivariate analysis, the number of brain metastases prior to radiosurgery was an independent prognostic factor (risk ratio, 2.46; 95\% confidence interval, 1.34-4.58; $\mathrm{P}=0.004)$.

\section{Discussion}

Brain metastases from lung cancer have become significant areas of focus in oncology research, including in genomic studies, and in the development of modern radiotherapy techniques and novel anticancer agents (12-14). A number of patients with few cerebral metastases receive radiosurgery, either alone or in combination with whole-brain irradiation. It is not yet clear whether the addition of whole-brain irradiation provides significant benefits for the patients. Additional whole-brain irradiation results in improved intracerebral control when compared with radiosurgery alone. The intracerebral control rates at 1 year were $66 \%$ after radiosurgery plus whole-brain irradiation and $51 \%$ after radiosurgery alone $(\mathrm{P}=0.015)$, respectively, in a retrospective study of 144 patients from Germany and the Netherlands who presented with brain metastases from various primary tumor types (5). In a randomized trial of 132 patients from Japan, the intracerebral failure rates at 1 year were $47 \%$ after radiosurgery plus whole-brain irradiation and $76 \%$ after radiosurgery alone $(\mathrm{P}<0.001)$ (4). Furthermore, in a prospective study from Japan re-evaluating 92 patients from the randomized trial, the rates of preservation of the neurocognitive function at 1 and 2 years were 79 and $79 \%$, respectively, following radiosurgery plus whole-brain irradiation compared with 53 and $43 \%$, respectively, following radiosurgery alone (7). In contrast to these results, a small randomized study $(n=58)$ found that a significant decline in neurocognitive functions was more frequent at 4 months after radiosurgery combined with whole-brain irradiation compared with after radiosurgery alone (8). However, this trial has been criticized, as neurocognitive functions were not evaluated at 12 months, when intracerebral control was significantly worse in the radiosurgery alone group (27 vs. 73\%; $\mathrm{P}<0.001$ ). Worse intracerebral control would likely have had a negative impact on the neurocognitive functions $(6,7)$. Despite the aforementioned study results, a number of physicians have reservations regarding additional whole-brain irradiation. This hesitation is also supported the fact that a retrospective study and a randomized trial showed that improvement in intracerebral control did not translate into significantly better survival $(4,5)$. The decision to add whole-brain irradiation to radiosurgery would be made easier in patients with a high risk of developing new cerebral metastases outside those treated with radiosurgery. This risk could be estimated if significant prognostic factors were identified.

In the current study, 10 factors were evaluated for significant associations with freedom from new brain metastases in lung cancer patients. The number of cerebral lesions was the only factor that had such an association. Patients with 2 lesions had a significantly greater risk than those patients with only a single lesion. Since the rates of freedom from new brain metastases at 1 and 2 years after radiosurgery alone were quite low in these patients, they are good candidates for the addition of whole-brain irradiation to radiosurgery. This will also likely apply to patients with $>2$ cerebral lesions. In contrast to patients with 1 or 2 cerebral lesions from lung cancer, those patients with $\geq 3$ lesions from lung cancer already receive radiosurgery plus whole-brain irradiation as the standard treatment procedure in the institutions that contributed to the present study. Therefore, patients with $\geq 3$ lesions were not available for the study.

In conclusion, freedom from new brain metastases was significantly associated with the number of cerebral lesions (1 vs. 2) prior to radiosurgery. Given the high rates of new cerebral lesions in patients with 2 brain metastases, these patients should be strongly considered for the addition of whole-brain irradiation to radiosurgery.

\section{References}

1. Marvaso G, Barone A, Vaccaro C, et al: Repeat stereotactic radiosurgery in the management of brain metastases from NSCLC: A case report and review of the literature. Oncol Lett 6: 897-900, 2013.

2. Rades D, Kueter JD, Veninga T, Gliemroth J and Schild SE: Whole brain radiotherapy plus stereotactic radiosurgery (WBRT+SRS) versus surgery plus whole brain radiotherapy (OP+WBRT) for 1-3 brain metastases: Results of a matched pair analysis. Eur J Cancer 45: 400-404, 2009.

3. Rades D, Veninga T, Hornung D, Wittkugel O, Schild SE and Gliemroth J: Single brain metastasis: Whole-brain irradiation plus either radiosurgery or neurosurgical resection. Cancer 118: 1138-1144, 2012.

4. Aoyama H, Shirato H, Tago M, et al: Stereotactic radiosurgery plus whole-brain radiation therapy vs stereotactic radiosurgery alone for treatment of brain metastases: A randomized controlled trial. JAMA 295: 2483-2491, 2006.

5. Rades D, Kueter JD, Hornung D, et al: Comparison of stereotactic radiosurgery (SRS) alone and whole brain radiotherapy (WBRT) plus a stereotactic boost (WBRT+SRS) for one to three brain metastases. Strahlenther Onkol 184: 655-662, 2008.

6. Meyers CA, Smith JA, Bezjak A, et al: Neurocognitive function and progression in patients with brain metastases treated with whole-brain radiation and motexafin gadolinium: Results of a randomized phase III trial. J Clin Oncol 22: 157-165, 2004. 
7. Aoyama H, Tago M, Kato N, et al: Neurocognitive function of patients with brain metastasis who received either whole brain radiotherapy plus stereotactic radiosurgery or radiosurgery alone. Int J Radiat Oncol Biol Phys 68: 1388-1395, 2007.

8. Chang EL, Wefel JS, Hess KR, et al: Neurocognition in patients with brain metastases treated with radiosurgery or radiosurgery plus whole-brain irradiation: A randomised controlled trial. Lancet Oncol 10: 1037-1044, 2009.

9. Rice DC: The staging of lung cancer. In: Diagnostic Pathology of Pleuropulmonary Neoplasia. Weissferdt A and Moran CA (eds): Springer, New York, NY,pp49-51, 2013.

10. Rades D, Huttenlocher S, Dahlke M, et al: Comparison of two dose levels of stereotactic radiosurgery for 1-3 brain metastases from non-small lung cancer. Anticancer Res 34: 7309-7313, 2014.
11. Kaplan EL and Meier P: Nonparametric estimation from incomplete observations. J Am Stat Assoc 53: 457-481, 1958.

12. Liang J, Liu X, Yin B, Liu H, Xiao J and Li Y: Efficacy assessment of pemetrexed treatment of an NSCLC case with brain metastasis. Oncol Lett 4: 1119-1121, 2012.

13. Lee HW, Seol HJ, Choi YL, et al: Genomic copy number alterations associated with the early brain metastasis of non-small cell lung cancer. Int J Oncol 41: 2013-2020, 2012.

14. Yang G, Wang Y, Wang Y, Lin S and Sun D: CyberKnife therapy of 24 multiple brain metastases from lung cancer: A case report. Oncol Lett 6: 534-536, 2013. 